\title{
Poly-Harmonic Distortion Model Extraction in Charge-Controlled One-Port Devices
}

\author{
Teresa M. Martín-Guerrero, José T. Entrambasaguas, Carlos Camacho-Peñalosa \\ Departamento de Ingeniería de Comunicaciones. E.T.S. Ingeniería de Telecomunicación \\ Universidad de Málaga, Andalucía Tech \\ Málaga (Spain) \\ \{teresa,jtem, ccp\}@ic.uma.es
}

\begin{abstract}
A charge-controlled, one-port device is used to describe and discuss the extraction procedure of a Poly-Harmonic Distortion (PHD) model in detail. For this case, both voltage and current waveforms are shown to be enough to fully characterize the PHD model. It is also shown that all the information specifically required for this PHD model definition can be stored in the Fourier coefficients of the incremental conductance and capacitance. The results are validated by comparing them with those obtained using a commercial circuit simulation tool.
\end{abstract}

Keywords - large-signal model; PHD model; charge-controlled model; X-parameters.

\section{INTRODUCTION}

In recent years, the characterization of non-linear devices in the frequency domain has been carried out using NVNA to characterize devices in terms of models based on X-parameters [1], S-functions [2], etc. These models have been described as a generalized version of conventional small-signal $\mathrm{S}$ parameters and are based on the Poly-Harmonic Distortion (PHD) principle [3]. They represent the non-linear response of a device to a large signal periodic excitation and the sensitivity of the different harmonics in response to changes in the harmonic content of the excitation. Incident waves at device ports are considered as the excitation signal while reflected waves are the response. The usefulness of and interest in these models have been proven and they are extensively used in the design of non-linear microwave circuits. They require highperformance specific measurement set-ups such as those based on the PNA-X family from Keysight. The main CAD tools have also included toolboxes that import X-parameters or Sfunctions into their schematics and, conversely, they can obtain these parameters from their non-linear simulation tools.

At the same time, charge-controlled equivalent circuits have been used extensively when modelling active solid state devices using compact models. The physical behaviour of these devices is properly represented by means of a current source shunted connected to a charge source (Fig. 1). These models are particularly useful in non-linear environments [4]. However they have two main limitations:

- At most, they can properly represent the intrinsic behaviour of the devices. For this reason the extrinsic shell should be de-embedded in order to extract the model properly.

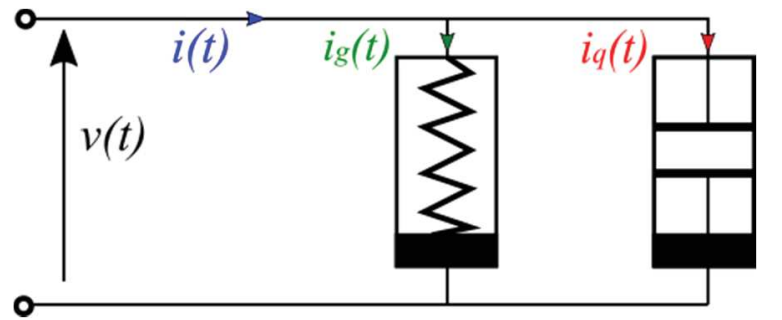

Fig. 3. Non-linear equivalent circuit for one port devices based on the charged-controlled model.

- These models are a quasi-static approach to the actual behaviour of the device. If non-quasi-static phenomena are noticeable, which happens at high frequencies, the model becomes no longer valid.

In this contribution, PHD model parameters, in particular Xparameters, and charge-controlled quasi-static models have been linked. It is shown that X-parameters can be calculated from I-V waveforms provided the device can be simulated by means of a charge-controlled model. In order to simplify the formulation, a one-port device (ideal diode) is used throughout the experiment. The extraction procedure is validated by comparing it with the X-parameters obtained using a commercial CAD tool (NI-AWR Design Environment).

\section{PHD MODELS FOR CHARGED-CONTROLlED ONE-PoRT DEVICES}

The so-called PHD principle [3] applies to non-linear devices driven by a large single-tone input signal. Because of the non-linearities, the response of the device will be a periodic signal with multiple harmonics. If the input signal is perturbed by the addition of small tones at different harmonics, the response can be linearly perturbed by additional harmonics at the output port. The sensitivity matrix represents how any small harmonic perturbation at the input port affects, by superposition, the harmonics at the output port.

If incident and scattered waves are used as variables, the PHD model can be written as follows:

$$
\mathbf{B}=\left.F^{X}(\mathbf{A}) \quad P H D \rightarrow \quad \mathbf{B} \cong \mathbf{B}\right|_{A_{1}, D C}+\left.\left[\frac{\partial \mathbf{b}}{\partial \mathbf{a}}\right]\right|_{A_{1}, D C} \cdot \mathbf{a}
$$


where $\mathbf{A}$ and $\mathbf{B}$ are the vectors that contain harmonics of both incident and scattered waves up to order $\mathrm{N}$, defined as:

$$
\begin{aligned}
\mathbf{B} & =\left[B_{-N}, B_{-N+1}, \ldots, B_{-1}, B_{0}, B_{1}, \ldots, B_{N-1}, B_{N}\right] \\
\mathbf{A} & =\left[A_{-N}, A_{-N+1}, \ldots, A_{-1}, A_{0}, A_{1}, \ldots, A_{N-1}, A_{N}\right] \\
\mathbf{b} & =\left[b_{-N}, b_{-N+1}, \ldots, b_{-1} b_{0}, b_{1}, \ldots, b_{N-1}, b_{N}\right] \\
\mathbf{a} & =\left[a_{-N}, a_{-N+1}, \ldots, a_{-1}, a_{0}, a_{1}, \ldots, a_{N-}, a_{N}\right]
\end{aligned}
$$

In the most frequent case $\mathbf{A}=\left[0, . .0, \mathrm{~A}_{1}, 0, \ldots 0\right]$, with $\mathrm{A}_{1}$ real (phase $=0$ ). $F^{X}$ is the non-linear functional relationship that describes the device and gives the relationship between the input wave $\mathbf{A}$ and the response wave $\mathbf{B}$. Vectors $\mathbf{a}$ and $\mathbf{b}$ represent the complex amplitude of the harmonics of the perturbation signals. The matrix $\left.\left[\frac{\partial \mathbf{b}}{\partial \mathbf{a}}\right]\right|_{A_{1}, D C}$ is the sensitivity matrix and includes the so-called $\mathbf{X}^{\mathbf{T}}$ (or $\mathbf{T}$ ) and $\mathbf{X}^{\mathbf{S}}$ (or $\mathbf{S}$ ) matrices, subsets of X-parameters. Vector $\mathbf{B}=\left.\mathbf{B}\right|_{A_{1}, D C}$ corresponds to the subset $\mathbf{X}^{\mathbf{F}}$ (or $\mathbf{F}$ ) of the $\mathrm{X}$-parameters. In this contribution the complete sensitivity matrix is just called the $\mathbf{X}$ matrix. It will be demonstrated in Section III how matrices $\mathbf{X}^{\mathbf{S}}$ and $\mathbf{X}^{\mathbf{T}}$ can be easily extracted from this unique $\mathbf{X}$ matrix, if required.

Matrices $\mathbf{X}^{\mathbf{S}}$ and $\mathbf{X}^{\mathbf{T}}$ verify that

$\left[b_{1}, b_{2}, \ldots, b_{N}\right]=\mathbf{X}^{\mathbf{S}}\left[a_{1}, a_{2}, \ldots, a_{N}\right]+\mathbf{X}^{\mathbf{T}}\left[a_{-1}, a_{-2}, \ldots, a_{-N}\right]$

where $\quad a_{-k}=a_{k}^{*} \quad b_{-k}=b_{k}^{*}$

The PHD model can also be defined using different circuit variables. It is still assumed that the device is driven by the same monochromatic signal $\mathbf{A}=\left[0, . .0, A_{1}, 0, \ldots 0\right]$. If the voltage is considered to be the input signal and the current is the response, the PHD model can be described as:

$$
\begin{aligned}
& \begin{array}{l}
\mathbf{I}=\left.F^{Y}(\mathbf{V}) \quad P H D \rightarrow \quad \mathbf{I} \cong \mathbf{I}\right|_{A_{1}, D C} \\
\mathbf{I}=\left[I_{-N}, I_{-N+1}, \ldots, I_{-1}, i_{0}, I_{1}, \ldots, I_{N-1}, I_{N}\right]
\end{array} \\
& \mathbf{V}=\left[V_{-N}, V_{-N+1}, \ldots, V_{-1}, V_{0}, V_{1}, \ldots, V_{N-1}, V_{N}\right] \\
& \mathbf{i}=\left[i_{-N}, i_{-N+1}, \ldots, i_{-1}, i_{0}, i_{1}, \ldots, i_{N-1}, i_{N}\right] \\
& \mathbf{v}=\left[v_{-N}, v_{-N+1}, \ldots, v_{-1,} v_{0}, v_{1,}, \ldots, v_{N-1}, v_{N}\right]
\end{aligned}
$$

where $F^{Y}$ is the non-linear functional relationship of the device, $\mathbf{V}$ and $\mathbf{I}$ are vectors containing the harmonics up to order $\mathrm{N}$ of both input and output signals. $\left.\left[\frac{\partial \mathbf{i}}{\partial \mathbf{v}}\right]\right|_{A_{1}, D C}$ is the new sensitivity matrix that represents how perturbations in the harmonics of the input signal (v) produce perturbations in the harmonics of the output signals (i). In the following, this matrix is called the $\mathbf{Y}$ matrix or admittance matrix.

If the device can be modelled by means of a chargecontrolled quasi-static equivalent circuit, the current can be split into two contributions:

$$
i(t)=i_{g}(v(t))+i_{q}(v(t))=i_{g}(v(t))+\frac{d}{d t}(q(v(t)))
$$

where $i_{g}(v(t))$ is the conductive current and $i_{q}(v(t))$ is the displacement current, and $q(v(t))$ is the charge source. This corresponds to an equivalent circuit like that detailed in Fig. 1. Using this definition in the time-domain, the sensitivity can be calculated as follows:

$$
\begin{aligned}
& {\left[\frac{\partial i}{\partial v}\right]=\frac{\partial i_{g}(v)}{\partial v}+\frac{d}{d t}\left[\frac{\partial q(v)}{\partial v}\right]=g(v)+\frac{d}{d t}[c(v)]} \\
& \text { with } \quad g(v)=\frac{\partial i_{q}}{\partial v} \quad c(v)=\frac{\partial q}{\partial v}
\end{aligned}
$$

The small-signal component of the current is given by:

$$
\tilde{i}=\left[\frac{\partial i}{\partial v}\right] \tilde{v}=\left(g(v)+\frac{d}{d t}[c(v)]\right) \tilde{v}
$$

and therefore it can be computed, in the frequency domain, using the implied convolution.

In the frequency domain the current can be expressed as:

$$
\left.\mathbf{I} \cong \mathbf{I}\right|_{A_{1}, D C}+\mathbf{Y} \cdot \mathbf{v} \text { with } \mathbf{Y}=\left.\left[\frac{\partial \mathbf{i}}{\partial \mathbf{v}}\right]\right|_{A_{1}, D C}=\mathbf{G}_{\mathbf{c}}+j \boldsymbol{\Omega} \cdot \mathbf{C}_{c}
$$

where $\mathbf{G}_{\mathbf{C}}$ and $\mathbf{C}_{\mathbf{C}}$ are the circulant matrices obtained from vector $\mathbf{G}$ and $\mathbf{C}$, respectively. Vectors $\mathbf{G}$ and $\mathbf{C}$ are the coefficients of the Fourier Transform of both the incremental conductance $(g(v(t)))$ and capacitance $(c(v(t)))$ functions, namely,

$$
\begin{aligned}
& \mathbf{G}=F T[g(v)]=\left[G_{-2 N}, G_{-2 N+1}, \ldots, G_{1}, G_{0}, G_{1}, \ldots, G_{2 N-1}, G_{2 N}\right] \\
& \mathbf{C}=F T[c(v)]=\left[C_{-2 N}, C_{-2 N+1}, \ldots, C_{1}, C_{0}, C_{1}, \ldots, C_{2 N-1}, C_{2 N}\right]
\end{aligned}
$$

Matrix $\Omega$ is diagonal and contains the harmonic frequencies. This matrix (actually $\mathrm{j} \Omega$ ) comes from the time-derivative. It should be noted that double amount of harmonics have to be considered $(2 \mathrm{~N})$ for $\mathbf{G}$ and $\mathbf{C}$ in order to calculate the convolution implied in (7) and avoid aliasing properly.

For the sake of clarity, the case for $\mathrm{N}=3$ with no displacement current $(q(t)=0)$ is illustrated in (9). Here $\mathbf{Y}=\mathbf{G}_{\mathbf{C}}$.

$\left[\begin{array}{c}i_{-6} \\ i_{-5} \\ i_{-4} \\ i_{-3} \\ i_{-2} \\ i_{-1} \\ \hline i_{0} \\ \hline i_{1} \\ i_{2} \\ i_{3} \\ i_{4} \\ i_{5} \\ i_{6}\end{array}\right]=\left[\begin{array}{lllllll|l|llllll}G_{0} & G_{-1} & G_{-2} & G_{-3} & G_{-4} & G_{-5} & G_{-6} & G_{6} & G_{5} & G_{4} & G_{3} & G_{2} & G_{1} \\ G_{1} & G_{0} & G_{-1} & G_{-2} & G_{-3} & G_{-4} & G_{-5} & G_{-6} & G_{6} & G_{5} & G_{4} & G_{3} & G_{2} \\ G_{2} & G_{1} & G_{0} & G_{-1} & G_{-2} & G_{-3} & G_{-4} & G_{-5} & G_{-6} & G_{6} & G_{5} & G_{4} & G_{3} \\ G_{3} & G_{2} & G_{1} & G_{0} & G_{-1} & G_{-2} & G_{-3} & G_{-4} & G_{-5} & G_{-6} & G_{6} & G_{5} & G_{4} \\ G_{4} & G_{3} & G_{2} & G_{1} & G_{0} & G_{-1} & G_{-2} & G_{-3} & G_{-4} & G_{-5} & G_{-6} & G_{6} & G_{5} \\ G_{5} & G_{4} & G_{3} & G_{2} & G_{1} & G_{0} & G_{-1} & G_{-2} & G_{-3} & G_{-4} & G_{-5} & G_{-6} & G_{6} \\ \hline G_{6} & G_{5} & G_{4} & G_{3} & G_{2} & G_{1} & G_{0} & G_{-1} & G_{-2} & G_{-3} & G_{-4} & G_{-5} & G_{-6} \\ \hline G_{-6} & G_{6} & G_{5} & G_{4} & G_{3} & G_{2} & G_{1} & G_{0} & G_{-1} & G_{-2} & G_{-3} & G_{-4} & G_{-5} \\ G_{-5} & G_{-6} & G_{6} & G_{5} & G_{4} & G_{3} & G_{2} & G_{1} & G_{0} & G_{-1} & G_{-2} & G_{-3} & G_{-4} \\ G_{-4} & G_{-5} & G_{-6} & G_{6} & G_{5} & G_{4} & G_{3} & G_{2} & G_{1} & G_{0} & G_{-1} & G_{-2} & G_{-3} \\ G_{-3} & G_{-4} & G_{-5} & G_{-6} & G_{6} & G_{5} & G_{4} & G_{3} & G_{2} & G_{1} & G_{0} & G_{-1} & G_{-2} \\ G_{-2} & G_{-3} & G_{-4} & G_{-5} & G_{-6} & G_{6} & G_{5} & G_{4} & G_{3} & G_{2} & G_{1} & G_{0} & G_{-1} \\ G_{-1} & G_{-2} & G_{-3} & G_{-4} & G_{-5} & G_{-6} & G_{6} & G_{5} & G_{4} & G_{3} & G_{2} & G_{1} & G_{0}\end{array}\right] *\left[\begin{array}{c}0 \\ 0 \\ v_{-2} \\ \end{array}\right.$

In (9) some sub-matrices have been highlighted. The yellow ones relate harmonics with subscripts with the same sign in the 
input and output spectra. They are usually called $\mathbf{Y}^{\mathbf{S}}$ in the bibliography [5], [6] through the analogy with the X-parameter subsets. The blue ones relate harmonics with subscripts with different signs and are usually called $\mathbf{Y}^{\mathbf{T}}$. It is evident from this simple case how harmonics from $-2 \mathrm{~N}$ to $2 \mathrm{~N}$ are required for the conductance function $\mathbf{G}$ (the same applies to $\mathbf{C}$ in a more general case).

It is worth highlighting that the information required to obtain the $\mathbf{Y}$ matrix are both current and voltages waveforms, with no additional specific measurements for the sensitivity matrix. All the information required for the calculation of the sensitivities can be stored in a couple of $\mathbf{G}$ and $\mathbf{C}$ vectors. Note that current should be split in its two conduction and displacement components. Furthermore, the formulation in terms of this $\mathbf{Y}$ matrix has the conductive and capacitance effects separated. This fact provides a compact model ready to be frequency scaled (as long as the quasi-static approach applies) [8].

\section{OBtAINING X PARAMETERS From THE AdMitTANCE Y MATRIX}

In this section the calculation of conventional X-parameters from the $\mathbf{Y}$ matrix is carried out. Firstly the $\mathbf{X}$ matrix will be obtained and, secondly, the so-called $\mathbf{X}^{\mathbf{S}}, \mathbf{X}^{\mathbf{T}}$ and $\mathbf{X}^{\mathbf{F}}$ will be extracted.

Taking into account the relationship between currents and voltages and incident and scattered waves [5], [6] it is straightforward to demonstrate that:

$$
\mathbf{X}^{\mathbf{F}}=\left.\mathbf{B}\right|_{A_{1}, D C}=\left.\frac{\mathbf{V}-Z_{0} \mathbf{I}}{2}\right|_{A_{1}, D C} \quad \mathbf{X}=\left[\mathbf{U}+Z_{0} \mathbf{Y}\right]^{-1} \cdot\left[\mathbf{U}-Z_{0} \mathbf{Y}\right](10)
$$

where $\mathbf{X}$ is the complete sensitivity matrix (as defined in Section II), $\mathbf{Y}$ is the admittance matrix defined in (7), $\mathbf{U}$ is the identity matrix and $Z_{0}$ is the reference impedance. $\mathbf{X}^{\mathbf{F}}$ is a subset of X-parameters that can be easily obtained from $\mathbf{V}$ and $\mathbf{I}$ using (10).

Once the $\mathbf{X}$ matrix is available, $\mathbf{X}^{\mathbf{S}}$ and $\mathbf{X}^{\mathbf{T}}$ can be extracted from it. Again, by using an example with $\mathrm{N}=3$, the $\mathbf{X}$ matrix becomes:
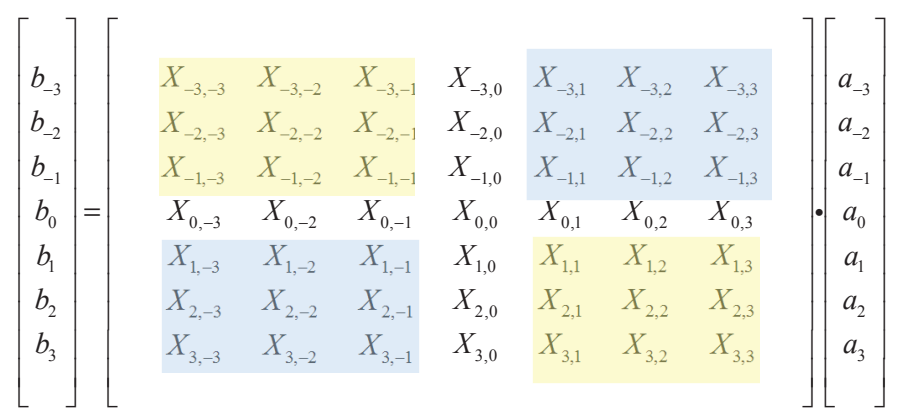

(11)

Again $\mathbf{X}^{\mathbf{S}}$ (the relationship between harmonics with subscripts with same sign) has been highlighted in yellow. $\mathbf{X}^{\mathbf{T}}$ (relating harmonics with subscripts with different signs) has been marked in blue.

In the general case ( $\mathrm{N}$ harmonics) both matrices can be extracted using (12). Although the use of $\mathbf{X}^{\mathbf{S}}$ and $\mathbf{X}^{\mathbf{T}}$ is quite extended, the proposed formulation, using just one $\mathbf{X}$ matrix, is more compact, more complete and it is believed to facilitate the understanding of the physical meaning of non-linear frequency domain models based on the PHD principle.

$$
\begin{aligned}
\mathbf{X}^{\mathbf{s}} & =\left[\begin{array}{cccc}
X(N+2, N+2) & X(N+2, N+3) & \ldots & X(N+2,2 N+1) \\
X(N+3, N+2) & X(N+3, N+3) & \ldots & X(N+3,2 N+1) \\
\ldots & \ldots & \ldots & \ldots \\
X(2 N+1, N+2) & X(2 N+1, N+3) & \ldots & X(2 N+1,2 N+1)
\end{array}\right] \\
\mathbf{X}^{\mathbf{T}} & =\left[\begin{array}{cccc}
X(N+2, N) & X(N+2, N-1) & \ldots & X(N+2,1) \\
X(N+3, N) & X(N+3, N-1) & \ldots & X(N+3,1) \\
\ldots & \ldots & \ldots & \ldots \\
X(2 N+1, N) & X(2 N+1, N-1) & \ldots & X(2 N+1,1)
\end{array}\right]
\end{aligned}
$$

\section{RESUltS}

An ideal diode (i.e., with no parasitic effects at all) has been used to illustrate the proposed extraction procedure. This device guarantees the proper use of the quasi-static charge-controlled model. It is described using the DIODE1 and PNCAP NI-AWR models for the non-linear conductance and capacitance, respectively. The model parameters are detailed in Table I.

TABLE I.
\begin{tabular}{|c|c|c|c|c|c|}
\hline \multicolumn{2}{|c|}{ DIODE 1} & \multicolumn{4}{c|}{ PNCAP } \\
\hline $\boldsymbol{I}_{\boldsymbol{0}}(\boldsymbol{m} \boldsymbol{A})$ & $\eta \boldsymbol{k}_{\boldsymbol{B}} \boldsymbol{T}(\mathbf{C V})$ & $\boldsymbol{C}_{\boldsymbol{J} \boldsymbol{0}}(\boldsymbol{p} \boldsymbol{F})$ & $\boldsymbol{V}_{\boldsymbol{J}}(\boldsymbol{V})$ & $\boldsymbol{M}$ & $\boldsymbol{F C}$ \\
\hline $4.610^{-5}$ & $4.510^{-21}$ & 1.8 & 0.5 & 0.5 & 0.5 \\
\hline
\end{tabular}

The device is driven by a source@1 GHz and biased @ 0 $\mathrm{V}$. The available power of the source $\left(\mathrm{Z}_{\mathrm{g}}=50 \Omega\right)$ is $6 \mathrm{dBm}$. The diode is analyzed using Harmonic Balance in a commercial tool (NI-AWR Design Environment). Voltage and current waveforms and their harmonics up to $\mathrm{N}=30$ have been calculated and exported to be used in the calculation of both $\mathbf{Y}$ and $\mathbf{X}$ matrices.

Fig. 2 represents the device I-V load-curve, including both conduction and displacement components. Both conductance and capacitance as a function of time have been calculated from (13). These functions have been used to build the $\mathbf{Y}$ matrix using (7). This matrix has been transformed into $\mathbf{X}$ using (10). Vector $\mathbf{X}^{\mathbf{F}}$ IQ has been obtained from $\mathbf{I}$ and $\mathbf{V}$ (see (10)) and matrices $\mathbf{X}^{\mathbf{S}_{\mathbf{I Q}}}$ and $\mathbf{X}^{\mathbf{T}} \mathbf{I Q}$ have been extracted from $\mathbf{X}$ using (12).

Furthermore, the PHD Model Generator (wizard included in NI-AWR Design Environment) has been used to calculate the $\mathrm{X}$-parameters (up to order 15, the maximum available) for the same device under the same frequency, load, biasing and input power conditions. The PHD Model Generator calculates Xparameters with a numerical procedure that emulates that used by NVNA. The simulation output file contains the $\mathbf{X}^{\mathbf{F}}$ AWR vector, and the $\mathbf{X}^{\mathbf{S}}{ }_{\text {AWR }}$ and $\mathbf{X}^{\mathbf{T}}{ }_{\text {AWR }}$ matrices.

In order to compare the $\mathbf{X}$ matrix obtained from the waveforms with that provided by the circuit simulator matrices, $\mathbf{X}^{\mathbf{S}_{\text {IQ }}}$ and $\mathbf{X}^{\mathrm{T}}$ IQ have been extracted for harmonics up to order 15. Fig. 3 details $\mathbf{X}^{\mathbf{S}}{ }_{\text {AWR }}$ and $\mathbf{X}^{\mathbf{S}}$ IQ. Fig. 4 is the difference between both matrices calculated as $\Delta^{S}(k, l)=X_{A W R}^{S}(k, l)-X_{I Q}^{S}(k, l)$. The mean error, given by $\overline{\left|\Delta^{S}(k, l)\right|}$, is $9.510^{-5}$. 


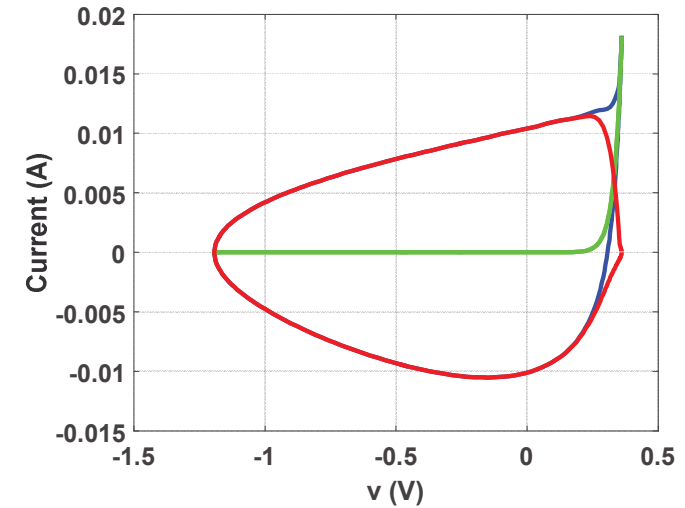

Fig. 2 Diode load-curve (blue). Conduction current $i_{g}$ (green) and displacement current $i_{q}=d q / d t$ (red) vs. input voltage $v$

Due to page limitations, the results for $\mathbf{X}^{\mathbf{T}}$ have not been included. They are qualitatively similar to those in Figs. 3 and 4. In this case the mean error, given by $\overline{\left|\Delta^{T}(k, l)\right|}$, is $5.810^{-5}$. The adjustment for $\mathbf{X}^{\mathbf{F}}$ does not deserve further comment because it is almost trivial, according to the definition (10). It can be concluded that the proposed formulation that calculates $\mathbf{X}$ parameters just from waveform data provides excellent results under ideal conditions.

\section{CONCLUSIONS}

It has been shown that PHD models such as X-parameters can be obtained just by using input-output waveforms (I-V) in the case of quasi-static, charge-controlled, one-port active devices. It has also been shown that the Fourier coefficients of the incremental conductance and capacitance constitute all the information required to fully characterize the PHD model. Moreover, the procedure described provides the non-linear admittance matrix that can be considered as a behavioural model closer to the physics of the device and potentially frequency scalable [8].

The proposed formulation has been applied to an ideal diode (a one-port device with no parasitic effects). The results obtained have been validated by comparing them with those provided by commercial software (NI AWR Design Environment).

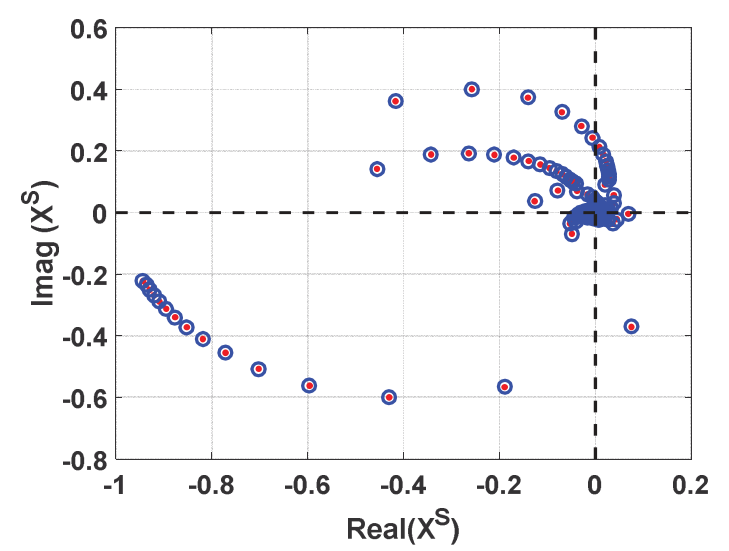

Fig. 3. $\quad \mathbf{X}_{\text {AWR }}^{\mathrm{S}}$ (red) and $\mathbf{X}_{{ }_{\mathrm{IQ}}}^{\mathrm{S}}$ (blue) up to 15 harmonics.

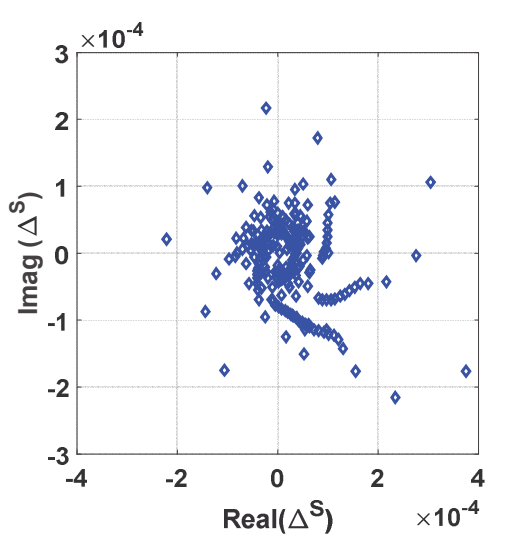

Fig. 4. $\quad \Delta^{S}(k, l)=X_{A W R}^{S}(k, l)-X_{I Q}^{S}(k, l)$. Difference between $\mathbf{X}_{\mathbf{A W R}}^{\mathbf{S}}$ and $\mathbf{X}^{\mathbf{S}}{ }_{\text {IQ }}$ up to 15 harmonics.

Despite the simplicity of this example, the extension to twoport devices is quite straightforward using this compact formulation in terms of just a single matrix. The application of this formulation to an actual device would require the proper de-embedding [4], [6] of the parasitic shell in order to reach the intrinsic plane in which the quasi-static approach could be valid.

\section{ACKNOWLEDGMENT}

The authors would like express their gratitude to Prof. José Carlos Pedro (University of Aveiro, Portugal) for sharing his knowledge with them. The authors also wish to thank the technical support of NI-AWR and National Instruments for providing software license in the frame of NI University Program.

This work has been supported by the Spanish Ministerio de Economía y Competitividad under Grant TEC2013-47106-C3-3-R and by the Junta de Andalucía under Grant TIC2012-1237.

\section{REFERENCES}

[1] D. E. Root, J. Verspecht, J. Horn, M. Marcu, Characterization, Modeling, and Design of Nonlinear RF and Microwave Components, Series: The Cambridge RF and Microwave Engineering Series. 2013.

[2] NMDG newsletter - IMS special edition 2009 [On-line: http://www.nmdg.be/newsletters/attach/SFunctions.pdf].

[3] J. Verspecht and D. E. Root, "Polyharmonic distortion modeling," in IEEE Microwave Magazine, vol. 7, no. 3, pp. 44-57, June 2006.

[4] D. Niessen, G. P. Gibiino, R. Cignani, A. Santarelli, D. M. M. P. Schreurs and F. Filicori, "Charge-Controlled GaN FET Modeling by Displacement Current Integration From Frequency-Domain NVNA Measurements," in IEEE Transactions on Microwave Theory and Techniques, vol. 64, no. 12, pp. 4382-4393, Dec. 2016.

[5] M. Fernandez-Barciela, A. M. Pelaez-Perez, S. Woodington, J. I. Alonso and P. J. Tasker, "Stretching the Design: Extending Analytical Circuit Design from the Linear to the Nonlinear Domain," in IEEE Microwave Magazine, vol. 15, no. 6, pp. 106-120, Sept.-Oct. 2014.

[6] R. Essaadali, A. Jarndal, A. B. Kouki and F. M. Ghannouchi, "A New GaN HEMT Equivalent Circuit Modeling Technique Based on XParameters," in IEEE Transactions on Microwave Theory and Techniques, vol. 64, no. 9, pp. 2758-2777, Sept. 2016.

[7] T. M. Martin-Guerrero, J. D. Baños-Polglase, C. Camacho-Peñalosa, M. Fernandez-Barciela, D. G. Morgan and P. J. Tasker, "Frequency domain-based approach for nonlinear quasi-static FET model extraction from large-signal waveform measurements," 2006 European Microwave Integrated Circuits Conference, Manchester, 2006, pp. 441-444.

[8] D. E. Root, R. M. Biernacki, M. Marcu, M. Koh and P. J. Tasker, "Frequency-scalable nonlinear behavioral transistor model from single frequency X-parameters based on time-reversal transformation properties," 2015 86th ARFTG Microwave Measurement Conference, Atlanta, GA, 2015, pp. 1-7. 